\title{
Kurzanalysen
}

Johannes Peters*

\section{Strategische maritime Herausforderungen an der NATO Nordflanke}

https://doi.org/10.1515/sirius-2021-4010

\section{Einleitung}

Mit dem Ende des Kalten Krieges gerieten die nordeuropäischen Gewässer aus dem maritim-strategischen Blickfeld des Westens im Allgemeinen und der NATO im Besonderen. Abgesehen von individuellen Sicherheitsüberlegungen einiger Anrainerstaaten, blieben die Seegebiete des Nordflankenraumes unterhalb des strategischen Radars. ${ }^{1}$ Die Ereignisse des Jahres 2014, der Krieg in der Ukraine, die völkerrechtswidrige Annexion der Krim sowie gesteigerte Aktivitäten der Russischen Marine im Nordatlantik und der Ostsee führten der Allianz die veränderten Realitäten vor Augen. Die Jahre der „Friedensdividende“ waren gezählt. Sie wurden abgelöst durch eine neue strategische Rivalität zwischen den westlichen (NATO) Staaten und, bezogen auf die Nordflanke, Russland. Diese wurde auf den NATO Gipfeln von Wales und Warschau erstmalig anerkannt und in den entsprechenden Erklärungen benannt. ${ }^{2}$

Der vorliegende Aufsatz befasst sich zunächst mit dem Begriff Nordflanke, seiner geographischen Definition und dessen Genese als vor allem taktisch-operativer Raum während des Kalten Krieges hin zu einem maritim-strategischen Konzept in der Gegenwart. Anschließend werden die maritim-strategischen Herausforderungen für das Bündnis benannt und Lösungsoptionen skizziert.

1 Pincus 2020, 53-54.

2 Pawlak 2021a, 163-164.

Article Note: Maßgebliche Arbeiten der letzten 1,5 Jahre stammen von Julian Pawlak. Der vorliegende Text orientiert sich inhaltlich und strukturell an seinen Arbeiten, welche in der Bibliografie aufgeführt sind.

*Kontakt: Johannes Peters, M.A., Leiter der Abteilung für maritime Strategie und Sicherheit am Institut für Sicherheitspolitik an der Universität Kiel, E-Mail: jpeters@ispk.uni-kiel.de

\section{Die Nordflanke - Definition und Begriffsgenese}

Etymologisch impliziert der Begriff Flanke das Vorhandensein eines Zentrums. Im hier relevanten militärischen Kontext bezeichnet er die Peripherie eines strategischen oder operativen Raumes. Heute subsummiert die NATO unter dem Begriff Nordflanke verschiedene Operationsräume: Die Nordsee und den Nordatlantik mit der Norwegischen Fjordsee, aber auch das flache Küstenmeer Ostsee mit seinen Zugängen über das Skagerrak und die Belte. ${ }^{3}$ Begrenzt wird dieser Raum durch drei strategische Zugänge oder Lücken (gaps). Im Westen durch das sogenannte GIUK gap, die gedachte Linie zwischen Grönland (Greenland), Island (Iceland) und dem Vereinigten Königreich (United Kingdom). Im Norden durch das Bear gap zwischen Spitzbergen und Norwegen und im Osten durch das Suwalki gap, den schmalen Landkorridor zwischen der russischen Exklave Kaliningrad und Belarus, welcher im Konfliktfall mit Russland das Nadelöhr für eine alliierte Verstärkung der baltischen Staaten über Land bildet. ${ }^{4}$

Historisch wurzelt der Begriff Nordflanke in der Annahme, dass ein konventioneller Krieg zwischen der NATO und dem Warschauer Pakt als Landkrieg in Zentraleuropa, und hier vor allem Westdeutschland, ausgetragen worden wäre. Das oben beschriebene Gebiet bildete somit die taktisch-operative Flanke im Norden, dessen strategische Bedeutung sich über die Jahrzehnte mehrfach änderte.

Ausschlaggebend für die Bedeutungsänderung waren technischer Fortschritt sowie die Perzeptionen der Strategien der jeweils gegnerischen Seite. Zu Beginn der Blockkonfrontation in den 1950er Jahren sahen die USA in Norwegen und der Norwegensee vor allem das Aufmarschgebiet für ihre taktischen und strategischen Bomberflotten. Von den nordnorwegischen Flugplätzen

3 Aufgrund der Spezifika dieses Seegebietes gibt es Argumente, es als gesonderten Operationsraum zu betrachten. Vgl.: Granholm 2021. Dies soll hier unterbleiben.

4 Pawlak 2021a, $164 \mathrm{ff}$. 
sollten, unterstützt von Flugzeugträgerverbänden, diese Bomber zunächst Ziele auf der Kola Halbinsel ausschalten, um dann tief ins sowjetische Kernland vorzustoßen. Mit dem Aufbau der sowjetischen U-Bootflotte wurde das Konzept der vorwärts dislozierten Trägerkampfgruppen ad acta gelegt und durch landgestützte Verbände in Nordnorwegen, Großbritannien und vor allem Island kompensiert. Gleichzeitig wurde eine Reihe passiver Sonaranlagen entlang der GIUK gap installiert, um das Vordringen sowjetischer U-Boote in den Nordatlantik zu überwachen, welche eine Bedrohung der lebenswichtigen transatlantischen Nachschubrouten darstellten. ${ }^{5}$

Mit der Entwicklung immer reichweitenstärkerer ballistischer Raketen und der Einführung von Atom U-Booten in den 1970er Jahren wurde die seegestützte nukleare Zweitschlagfähigkeit ein Eckpfeiler der sowjetischen Abschreckung. Entsprechend erfuhr die sowjetische Doktrin eine Anpassung. Das sogenannte bastion concept wurde entwickelt. Es war nun nicht mehr nötig, die ballistischen U-Boote weit westlich in den Nordatlantik zu entsenden. Das neue Operationsgebiet war die Barentssee bis etwa zum Bear gap und das Nordpolarmeer. Aus dieser sicheren Bastion nahe ihrer Heimathäfen auf der Kola Halbinsel heraus wären sie in der Lage gewesen, jedes Ziel in Nordamerika zu erreichen. Die sowjetische Strategie bestand in einer totalen Kontrolle dieser Seeregion (sea control), vornehmlich durch landgestützte Flugzeuge. Der Flotte kam die Aufgabe zu, der NATO die Nutzung der Norwegensee ab dem GIUK gap zu verwehren (sea denial). Prinzipiell ist dieses Konzept bis heute gültig. Um insbesondere den sowjetischen sea denial Plänen zu begegnen, entwickelte die NATO ab den 1980er Jahren wieder eine offensivere maritime Strategie. Bereits in der Frühphase eines Konfliktes sollte maximaler Druck auf die sowjetischen Bastionen ausgeübt werden, um die Nordflotte in der Norwegensee $\mathrm{zu}$ binden, und so ein Vordringen in den Nordatlantik $\mathrm{zu}$ verhindern. ${ }^{6}$ Zusammenfassend lässt sich festhalten, dass der Begriff Nordflanke nur insofern richtig ist, als die Region im Falle eines heißen Krieges zwischen den politischen Blöcken die Flanke des Hauptschlachtfeldes in Zentraleuropa dargestellt hätte. Bezogen auf den maritimen Anteil hätte sie aber das Zentrum der Auseinandersetzung gebildet.

5 Das berühmte Sound Surveillance System (SOSUS), siehe auch Tanner 2017, 8 ff.

6 Tamnes 2017, $12 \mathrm{ff}$.

\section{Entspannung und Annäherung}

Der Zusammenbruch des Warschauer Paktes und das damit einhergehende Ende des Kalten Krieges bedeuteten einen epochalen Wandel für den Nordflankenraum. Mit dem Beitritt der osteuropäischen Staaten zur NATO und zur Europäischen Union (EU) veränderte sich die Sicherheitsarchitektur grundlegend. 2004 waren sechs Anrainer der ehemals als „rote See“ bezeichneten Ostsee Mitglieder der NATO und alle bis auf Russland Teil der EU. Mit dem „Partnership for Peace“ (PfP) Programm der NATO ab 1994 schlug sich die politische Annährung auch militärischoperationell nieder. Das erste PfP Manöver (COOPERATIVE VENTURE 94) fand unter russischer Beteiligung symbolträchtig in der Norwegensee statt und auch beim jährlichen US geführten Ostseemanöver BALTIC OPERATIONS (BALTOPS) wurde die russische Marine ein regelmäßiger Gast - bis 2013. ${ }^{7}$

Mit den Zeichen auf Wandel und Annäherung, veränderten sich alliierte Marineoperationen grundlegend. In Ermangelung eines peer competitors stellte sich die Frage nach der zukünftigen Rolle der NATO und das Bündnis musste seine Rolle innerhalb der internationalen Sicherheitsarchitektur anpassen - oder alternativ selbst Geschichte werden. „Out of area or out of business“ war damals das Motto der Stunde.

NATO-Einheiten spielten nun eine wichtige Rolle bei der Konflikt- und Krisenbewältigung im östlichen Mittelmeer, dem Persischen Golf oder in den Gewässern um das Horn von Afrika. Ausgeführt wurden diese niedrigschwelligen Einsätze von hochentwickelten „Kalten Kriegern“, optimiert für das hochintensive Seegefecht und die U-Boot Jagd in den kalten Gewässern des Nordatlantiks. Je länger diese Situation anhielt, desto mehr passten sich Planer und Streitkräfte an: Manöver konzentrierten sich mehr auf humanitäre Einsätze und Katastrophenhilfe, oder auf Boarding-, Such- und Beschlagnahmeoperationen als auf Nachschubsicherung über den Atlantik oder U-Boot Jagd in der GIUK gap. Politiker aller NATO Mitgliedstaaten waren großzügig im Einlösen der Friedensdividende nach dem Ende des Kalten Krieges. Dies führte zu einem breiten Rückgang der Verteidigungsbudgets und einer erheblichen Verkleinerung der NATO Flotten. Heute verfügen alle westlichen Marinen über deutlich weniger Flaggenstöcke als $1990 .^{8}$

7 Pawlak 2021b.

8 Stöhs und Pawlak 2019, 243 ff. Sowie in großer Detailtiefe: Stöhs 2018. 
Doch nicht nur die Zahl von Marineschiffen änderte sich, auch deren Fähigkeitsprofil unterlag vielfältigen Wandlungen. Neue Plattformen sollten die Fülle niedrigschwelliger Aufgabenszenarien abdecken, mit denen sich westliche Marinen in den 1990er und frühen 2000er Jahren konfrontiert sahen. Um überhaupt Haushaltsmittel zu generieren, lautetet das Credo „Mehrrollenfähigkeit.“ Diese Entwicklung führte insbesondere in Europa zu relativ großen, aber im Vergleich zu ihren Vorgängern im Kalten Krieg leicht bewaffneten Fregatten, optimiert für lange Stehzeiten in weit entfernten Operationsräumen und den unteren Bereich des Intensitätsspektrums - die neueste Fregattenklasse F125 der Deutschen Marine kann hier als exemplarisch gelten.

In diesem Zusammenhang verkümmerten die Fähigkeiten und Fertigkeiten zum Führen des hochintensiven Seegefechts bei allen NATO-Marinen, wenn auch in unterschiedlichem Ausmaß. Aufgrund seines hochkomplexen Wesens und der Notwendigkeit, permanent an der Grenze des technischen Fortschritts zu operieren, gilt dies insbesondere für die ehemalige Kernkompetenz der Allianz die U-Boot Jagd. ${ }^{9}$

\section{Von Annäherung zur Konfronta- tion - 2014 als Zeitenwende}

Genau 20 Jahre nach OCEAN VENTURE erschütterten der Krieg in der Ukraine und die Annexion der Krim die europäische Sicherheitsarchitektur grundlegend. Anstelle von good order at sea und out of area rückten Landes-und Bündnisverteidigung wieder in den strategischen Fokus der NATO. Ausdruck fand dieser Paradigmenwechsel an der Nordflanke in der Enhanced Forward Presence im Baltikum und einer Refokussierung auf großangelegte Manöver wie BALTOPS, NORTHERN COST oder TRIDENT JUNCTURE 18 - der größten NATO Übung seit 1980. Doch während es auf den ersten Blick so aussieht, als dominierten NATO und EU Staaten die Seegebiete der Nordflanke, hält dieser Eindruck einer genaueren Betrachtung nicht stand. ${ }^{10}$ Russland hat sich wieder zu einem ernstzunehmenden Herausforderer entwickelt, der das Bündnis vor verschiedenste Herausforderungen stellt, welchen es nur kollektiv begegnen kann. Diese Herausforderung wird vor allem mit Blick auf die Unterwasserkriegsführung und die hybride Kriegsführung ersichtlich.

9 Peters 2021a, S. 94. 10 Pawlak 2021b.

\subsection{Russische Unterwasserkriegsführung}

Die russische Marine ging in den 1990er und frühen 2000er Jahren durch ein „Tal der Tränen“, mit dem Verlust der KURSK im Jahr 2000 als dramatischem Tiefpunkt. Die nuklear angetriebenen U-Boote, das traditionelle Herz der Flotte, konnten jedoch zumindest einen Teil ihrer Fähigkeiten und Plattformen erhalten. Gleiches galt für die entsprechenden Werft- und Entwicklungseinrichtungen. Obwohl die russische U-Boot-Flotte (nuklear und konventionell angetrieben) noch immer hauptsächlich aus modernisierten und aufgerüsteten Sowjetbeständen besteht, konnten einige bedeutende Fortschritte erzielt werden.

Mit den Jagd U-Booten der YASEN Klasse und den ballistischen Raketen U-Booten der BOREI Klasse hat Russland seine nuklear angetriebene U-Bootflotte auf zwei Klassen verschlankt und folgt damit dem Vorbild etwa der USA oder Großbritanniens. Beide Klassen haben Startschwierigkeiten und Kinderkrankheiten mittlerweile überwunden und gelten als hochentwickelte Boote und echte peer-competitor für westliche Marinen. Wann der Nachfolger der kürzlich aktualisierten dieselelektrischen KILO-Klasse, die KALINA-Klasse, welche mit einem außenluftunabhängigen Antriebssystem (AIP) ausgestattet werden soll, Einsatzreife hat, ist derzeit hingegen noch nicht absehbar. ${ }^{11}$ Entscheidender ist jedoch, dass es Russland gelungen ist, eine Serienproduktion für seine U-Boote zu etablieren. Somit können Erkenntnisse aus See-Erprobungen und Einsätzen direkt in den Produktionsprozess einfließen. Innovationszyklen werden dadurch kürzer und weniger vorhersehbar.

Besonders die Boote der YASEN Klasse gelten hinsichtlich ihres Sonar- und elektromagnetischen Profils denen der US Navy als ebenbürtig. Ausgestattet mit einem Senkrechtstartersystem können sie 32 Marschflugkörper tragen. Bewaffnet mit Kalibr- und/oder Onyx-Marschflugkörpern und vorbereitet auf die Aufnahme des in der Entwicklung befindlichen Hyperschall-Marschflugkörpers Tsirkon, sind diese Plattformen in eine umfassende nationale Sicherheitsstrategie eingebettet. Diese umfasst die Aufrüstung von See-, Luft-, Land- und Weltraumfähigkeiten in Kombination mit der Fähigkeit zum Führen von Präzisionsschlägen über große Entfernungen. Sie werden dadurch zu einer strategischen Herausforderung für die NATO und die westlichen Marinen. ${ }^{12}$

Doch nicht nur technisch, auch operativ versucht Russland an alte Größe anzuknüpfen. Im Oktober 2019 machte es internationale Schlagzeilen, als es nicht weniger als

11 Hicks 2016, $14 \mathrm{ff}$.

12 Nordenman und Stavridis 2019, $132 \mathrm{ff}$. 
zehn Atom-U-Boote gleichzeitig in die Nordnorwegensee und den Nordatlantik entsandte. Flankiert von der Übung OCEAN SHIELD in der Ostsee und kleineren Übungen im Mittelmeer löste dies Schockwellen über den Atlantik aus, da es den Westen völlig unvorbereitet traf. Obwohl Putin auch innenpolitische Ziele verfolgte, war es vor allem ein starkes strategisches Signal an die NATO und die USA, dass Russland in der Lage ist, die transatlantischen Seeverbindungswege in großem Umfang und fast ohne Vorwarnzeit zu stören. ${ }^{13}$

Wie bereits erläutert, war und ist eine Geleitzugschlacht im Nordatlantik nicht im Interesse Russlands. Seine U-Boot gestützten Marschflugkörper versetzen es in die Lage, europäische Seehäfen wie Bremerhaven oder Antwerpen, aus der relativen Sicherheit der Nordnorwegensee heraus zu bedrohen. Die NATO benötigt daher an der Nordflanke wieder die Mittel und Fähigkeiten zur U-Boot Jagd. ${ }^{14}$ Traditionelle U-Boot Jagd ist allerdings zeitaufwendig, plattformintensiv und extrem kostspielig. Ausgeführt werden muss sie von ausgedünnten Seestreitkräften, welche seit 2014 hart darum kämpfen, den jahrzehntelangen Rückbau in einen vorsichtigen Aufwärtstrend umzukehren. Vor dem Hintergrund der COVID19-Pandemie bleibt abzuwarten, ob diese Trendumkehr auf Sand gebaut ist oder nicht. ${ }^{15}$

Um dem Dilemma steigender Anforderungen vor dem Hintergrund fiskalischer Unsicherheit zu begegnen, müssen zukünftige U-Boot-Jagd Konzepte beides sein: erschwinglicher in Friedenszeiten und effektiver und besser skalierbar im Krieg. Sie werden daher auf unbemannte und autonome Systeme angewiesen sein. Der Entwicklung und dem Einsatz dieser Systeme kommt in den kommenden Jahrzehnten eine entscheidende Rolle zu. Dabei muss zwischen den drei Phasen der U-Boot Jagd unterschieden werden: Detektierung, Verfolgung und Bekämpfung. Die Geschichte zeigt, dass ein U-Boot, sobald es entdeckt wird, aufgrund seiner relativ geringen Geschwindigkeit und des Fehlens ausreichender Gegenmaßnahmen seinen taktischen Vorteil verliert. Ein erkanntes U-Boot wird sich höchstwahrscheinlich zurückziehen, anstatt auf Posten $\mathrm{zu}$ bleiben und anzugreifen. Daher muss den ersten beiden Schritten, Detektierung und Verfolgung, viel mehr Bedeutung beigemessen werden. Unbemannte Systeme bieten hier ein großes Potenzial für effektivere und relativ kostengünstige U-Boot Jagd Konzepte. ${ }^{16}$

13 Moss 2020, $38 \mathrm{ff}$.

14 Pawlak 2021b.

15 Peters 2021a, 103.

$16 \mathrm{Zu}$ den Möglichkeiten von unbemannten Systemen, Ihren Grenzen und den Notwendigkeiten von „manned-unmanned teaming“,

\subsection{Hybride Kriegsführung}

Unter hybrider Kriegsführung (oder dem im US-Amerikanischen Englisch gebräuchlicherem Begriff grey-zone warfare) versteht man die koordinierte und verzahnte offene wie verdeckte Anwendung von militärischen, paramilitärischen aber auch zivilen Mitteln, inklusive Cyberattacken unterhalb einer Schwelle zur kriegerischen Handlung. Die Kunst des Anwenders liegt dabei darin, diese Schwelle geschickt auszuloten und, für den Fall eines Überschreitens, nicht eindeutig als Verursacher benennbar zu sein. ${ }^{17}$ Wie Russland dieses Spiel geschickt spielt, soll im Folgenden verdeutlicht werden.

Parallel zur Modernisierung seiner Marine unternimmt Russland erhebliche Anstrengungen, in der Tiefsee operieren zu können. Das derzeit größte U-Boot weltweit, die 2019 in Dienst gestellte BELGOROD, ein umgebautes Atom U-Boot der OSCAR-II Klasse, wird als Testplattform für neue Unterwasserwaffen, vor allem aber als Mutterschiff für tief tauchende Kleinst-U-Boote fungieren. Ergänzt durch eine neue Klasse von Tiefseeforschungsschiffen zielt Russland vor allem auf Seekabel. Auf dem Meeresboden liegend, sind diese Kabel zu den Lebensadern der westlichen Welt im digitalen Zeitalter geworden. Etwa 99 Prozent der weltweiten Datenmengen werden durch sie übertragen. Im Frühstadium eines Konflikts hätte ihre Störung oder Unterbrechung hohe Priorität. Einen weiteren Angriffsvektor stellt das Anzapfen zur Informationsgewinnung dar. Bislang werden diese Kabel nicht als kritische Infrastruktur für westliche Sicherheit, Wohlstand und Wohlergehen gesehen, sondern als Privateigentum der Unternehmen, die sie betreiben. Der Schutz und nötigenfalls die Verteidigung dieser Infrastruktur muss aber in jeder westlichen maritimen Strategie hohe Priorität haben. Die Tatsache, dass die russischen Tiefseefähigkeiten nicht der Marine, sondern der Hauptdirektion für Tiefseeforschung (GUGI) angehören, macht ihren rechtlichen Status in einem Konflikt weniger klar und damit schwieriger zu adressieren. ${ }^{18}$

Von besonderer Bedeutung ist dies derzeit in der Ostsee. Aufgrund ihrer geographischen Enge und der Möglichkeit, jeden Punkt auf See mit landgestützten Systemen erreichen zu können, gelten klassische Seemachtkonzepte hier nur bedingt. Jüngste Entwicklungen deuten aber darauf hin, dass diese Einschränkungen absoluter Natur sind. Im Konfliktfall würde der See- und Luftraum der Ostsee durch elektromagnetische Maßnahmen und

sowie komparativen Kostenvorteilen siehe in großer Breite und Tiefe: Clark 2015.

17 Hoffman 2021, 69-71.

18 Ansari 2021, 16-18. 
Gegenmaßnahmen sofort zu einer Sperrzone, nicht nur für Überwasser-Kriegsschiffe und Kampfflugzeuge, sondern auch für die Kommunikation. Damit verbleibt als einziger möglicher Navigations-, Kommunikations- und Operationsraum die Wassersäule - sie wird zum Operationsgebiet. Die Partei, welche in einem Konfliktszenario diesen Raum zuerst und am besten nutzen kann, wird obsiegen. Um diese Kaltstartfähigkeit zu maximieren und sich einen Vorteil zu verschaffen, muss das nötige Wissen und die Infrastruktur bereits im Vorfeld eines Konfliktes erworben und installiert werden. Diese Sicht auf die Wassersäule als eigenständigen, neuen Operationsraum bezeichnet man als seabed warfare. Russland ist derzeit sehr aktiv dabei, dieses potenzielle zukünftige Schlachtfeld in der Ostsee zu seinen Gunsten zu gestalten. ${ }^{19}$

Vor diesem Hintergrund ist die kürzlich fertiggestellte Nord Stream 2 Pipeline, die von St. Petersburg bis zur deutschen Küste quer durch die Ostsee verläuft, eine Bedrohung nicht nur für die nationale Sicherheit Deutschlands, sondern auch für die Sicherheit der EU. Ein von einer Gruppe ukrainischer Experten veröffentlichter Artikel argumentiert, dass der Betrieb und die Wartung der Pipeline ausschließlich in den Händen des russischen Unternehmens Gazprom liegen. Dies beinhaltet alle Sensoren zur Messung z. B. der Durchflussmenge an Gas, sowie Glasfaserkabel zur Übertragung dieser Daten an Land. Dieses Sensor-Transmitter-System hat ein enormes Dual-Use-Potenzial. North Stream 2 habe das Potenzial, im Konfliktfall $\mathrm{zu}$ einer gigantischen Sensorkette unter russischer Kontrolle zu werden. Obwohl vor allem von internationaler Seite oft gewarnt, betonte die deutsche Politik bisher stets den ausschließlich kommerziellen Charakter des Pipelineprojektes und bestritt jegliche Sicherheitsauswirkungen für einzelne Länder oder die Region. Ob die zukünftige Regierung ähnlich zurückhaltend sein wird, bleibt abzuwarten. $^{20}$

\section{Mögliche Lösungsansätze}

Die Entwicklung und Proliferation von Marschflugkörpern mit großer Reichweite hat weite Teile der Weltmeere $\mathrm{zu}$ einem gefährlicheren Umfeld für Überwasserschiffe gemacht als je zuvor. Dieser Umstand und die Tatsache, dass U-Boote oft als Kräfteausgleich gegen eine überlegene Überwasserflotte angesehen werden, hat zu einem

19 Peters 2021b.

20 Peters 2021b, siehe auch Joachim Krause: Nord Stream 2 könnte zur Falle werden, Neue Zürcher Zeitung, 7.7.2021, 14.
Anstieg der weltweiten U-Boot-Beschaffung in den letzten Jahren geführt.

Aufgrund ihrer komplexen Natur war U-Boot Jagd schon immer ein teilstreitkraft- und streitkräftegemeinsames Unterfangen. Wie gezeigt, hat sich das bloße Rekurrieren auf Techniken und Taktiken des Kalten Krieges als unzureichend erwiesen, um den heutigen Herausforderungen zu begegnen. Angesichts langwieriger Beschaffungszyklen und den stetig steigenden Kosten für state of the art Plattformen, welche in den knappen Budgets der NATO Seestreitkräfte kaum noch abbildbar sind, müssen daher neue Ansätze gefunden werden. ${ }^{21}$

Um dem doing more with less Dilemma zu entkommen, werden NATO Marinen in Zukunft viel stärker auf unbemannte und autonome Systeme setzen müssen. Diese stellen der Allianz erschwingliche, flexible, schnell einsetzbare und skalierbare U-Boot Jagd Techniken und Taktiken zur Verfügung. Die Beschaffung von verfügbarer off the shelf-Technik würde es auch kleineren Marinen erlauben, einen bedeutenden Anteil zur alliierten U-Boot Jagd Fähigkeit beizusteuern.

Um das volle Potenzial unbemannter Systeme für die NATO zu entfalten, müssen daher einige Hausaufgaben gemacht werden:

- U-Boot Jagd muss wieder stärker beübt werden. Die jährlichen Manöver DYNAMIC MONGOOSE/MANTA müssen um eine dezidiert unbemannte Komponente ergänzt werden.

- Entwicklung, Implementierung und Beschaffung neuer Technologien müssen mit den Verbündeten koordiniert werden, um Interoperabilität und sichere Kommunikation zu gewährleisten. Das kürzlich gegründete Center for Maritime Research and Experimentation (CMRE) und das NATO Maritime Unmanned Systems Network (MUS) sind Schritte in die richtige Richtung. Die PESCO Initiative der EU könnte auf dieselbe Weise funktionieren.

- Es gibt politische Vorbehalte gegen unbemannte Systeme. Während Staaten wie die USA, GB oder Frankreich beispielsweise die Möglichkeiten und operativen Vorteile sehen, herrscht insbesondere in Deutschland breite Skepsis vor. Jeder Verbündete muss daher eine klare Position festlegen, inwieweit er unbemannte Systeme unterstützen kann und will und seine Rolle in einem alliierten U-Boot Jagd Szenario definieren..$^{22}$

$21 \mathrm{Zu}$ den Herausforderungen bei der Vereinbarung von Modernisierungen und „Techflation“ und den sich ergebenen Konsequenzen für die Flottenstruktur kleiner und mittlerer Marinen siehe: Stöhs 2021. 22 Peters 2021a, S. 107-108. 
Hybride Konfliktszenarien, durchgeführt von irregulären Kräften mit unklarer Staatszugehörigkeit und unterhalb der Schwelle zur kriegerischen Handlung gehalten, sind heute wahrscheinlicher als vor 30 Jahren. Um diesen Bedrohungen auf politischer Ebene zu begegnen, bedarf es zunächst der Anerkennung ihrer Existenz, sowie gemeinsamer Definitionen und Bewertungskriterien. Während U-Boote nicht notwendigerweise Teil eines hybriden Szenarios sein müssen, wird es die Wassersäule mit großer Sicherheit sein. Die NATO muss daher ihr Abschreckungskonzept erweitern.

Hybriden Bedrohungen kann aufgrund ihrer dargestellten Natur nur unzureichend mit konventioneller militärischer Abschreckung begegnet werden. Dennoch müssen sie sich in einem konsistenten, zukunftsorientierten Abschreckungskonzept wiederfinden. Im Kern hat Abschreckung zum Ziel, die politischen, ökonomischen und militärischen Kosten im Kalkül des potentiellen Gegners für bestimmte Handlungen so zu erhöhen, dass diese unattraktiv werden. Bei hybriden Bedrohungen, wie beispielsweise einem breit angelegten Cyberangriff, ist Resilienz daher eine Schlüsselgröße. Gemeint ist in erster Linie die technische Resilienz von Systemen (etwa von Systemen der unterseeischen Datenübertragung) sowie die Vermeidung von neu entstehenden Verwundbarkeiten gegen hybride Angriffe (etwa Nord Stream 2). Diese Debatte sollte nicht nur in Militärkreisen, sondern durchaus breiter geführt werden. Es bedarf auch einer gesellschaftlichen Resilienz. Hybride Kriegsführung hat eine gewichtige psychologische Komponente, welche darauf abzielt, den gesellschaftlichen Rückhalt der politischen Akteure zu schwächen. Je unwahrscheinlicher das Erreichen dieses Ziels ist, desto unattraktiver der hybride Angriff. Es bedarf daher einer breiteren politischen Debatte über hybride Risiken nicht nur bei Cyberattacken, sondern auch im maritimen Bereich.

\section{Literaturverzeichnis}

Ansari, Usman (2021): Worldwide net cable vulnerability opens new front in any future war, Warships International Fleet Review, (2), 16-18

Clark, Bryan (2015): The emerging era in undersea warfare. Washington, D.C.: Center for Strategic and Budgetary Assessments (CSBA)

Granholm, Niklas (2021): Like Butter Scraped Over Too Much Bread. The North Sea, Skagerrak and Kattegat - an overlooked region in maritime defence and security?, in: Pawlak/Peters 2021, 181-195

Hicks, Kathleen H. (2016): Undersea warfare in Northern Europe. Washington, D.C./Lanham, MD: Rowman \& Littlefield

Hoffman, Frank G. (2021): Icognito Hybrid Threats: Avoiding the Alliance's Trident, in: Pawlak/Peters 2021, 69-92

Moss, Richard A. (2020): Russia basks in cold war glory, in: US Naval Institute Proceedings, 146 (10), 38-43

Nordenman, Magnus/Stavridis, James (2019): The new Battle for the Atlantic. Emerging naval competition with Russia in the Far North. Annapolis, MD: Naval Institute Press

Pawlak, Julian (2021a): Bridge the Gaps - An Allied Naval Approach for Northern Europe, in: Pawlak/Peters 2021, 163-179

Pawlak, Julian (2021b): Turned Tides: The Northern Flank's Evolving Strategic Role from the Cold War to the 21st Century. Conference Paper, McMullen Naval History Symposium 2021, US Naval Academy, 23.09.2021

Pawlak, Julian/Peters, Johannes, Hrsg. (2021): From the North Atlantic to the South China Sea. Allied Maritime Strategy in the 21st Century. Kiel: ISPK (Seapower Series, 4)

Peters, Johannes (2021a): Below the Surface: Undersea warfare challenges in the 21st century, in: Pawlak/Peters2021, 93-110

Peters, Johannes (2021b): The evolving role of the German Navy in the underwater domain from the Cold War to the present. Conference Paper, McMullen Naval History Symposium 21, US Naval Academy, 23.09.2021

Pincus, Rebecca (2020): Towards a New Arctic. Changing Strategic Geography in the GIUK Gap, The RUSI Journal, 165 (3), 50-58

Stöhs, Jeremy (2018): The decline of European naval forces. Challenges to sea power in an age of fiscal austerity and political uncertainty. Annapolis, MD: Naval Institute Press Stöhs, Jeremy (2021): How High? The Future of European Naval Power and the High-End Challenge. Copenhagen: Djøf Publishing in cooperation with Centre for Military Studies

Stöhs, Jeremy/Pawlak, Julian (2019): Strategische Herausforderungen und Handlungsoptionen westlicher Politik im nördlichen Atlantik, SIRIUS - Zeitschrift für Strategische Analysen, 3 (3), 242-254

Tamnes, Rolf (2017): The Significance of the North Atlantic and the Norwegian Contribution, in: Olsen, John Andreas (Hrsg.): NATO and the North Atlantic. Revitalising collective defence, Royal United Services Institute, Whitehall Papers, 87 (1). London, Abingdon: Routledge Journals, 9-31 